\title{
European Risk Assessment Guidance for Infectious Diseases transmitted on Aircraft - the RAGIDA project
}

K Leitmeyer (katrin.leitmeyer@ecdc.europa.eu) ${ }^{1}$

1. European Centre for Disease Prevention and Control (ECDC), Stockholm, Sweden

Citation style for this article:

Leitmeyer K. European Risk Assessment Guidance for Infectious Diseases transmitted on Aircraft - the RAGIDA project. Euro Surveill. 2011;16(16):pii=19845

Available online: http://www.eurosurveillance.org/ViewArticle.aspx?Articleld=19845

In order to assist national public health authorities in the European Union to assess the risks associated with the transmission of infectious agents on board aircrafts, the European Centre for Disease Prevention and Control initiated in 2007 the RAGIDA project (Risk Assessment Guidance for Infectious Diseases transmitted on Aircraft). RAGIDA consists of two parts: the production of a systematic review and a series of disease-specific guidance documents. The systematic review covered over 3,700 peer-reviewed articles and grey literature for the following diseases: tuberculosis, influenza, severe acute respiratory syndrome (SARS), invasive meningococcal disease, measles, rubella, diphtheria, Ebola and Marburg haemorrhagic fevers, Lassa fever, smallpox and anthrax. In addition, general guidelines on risk assessment and management from international aviation boards and national and international public health agencies were systematically searched. Experts were interviewed on case-based events by standardised questionnaires. Disease-specific guidance documents on tuberculosis, SARS, meningococcal infections, measles, rubella, Ebola and Marburg haemorrhagic fevers, Lassa fever, smallpox and anthrax were the result of consultations of disease-specific expert panels. Factors that influence the risk assessment of infectious disease transmission on board aircrafts and decision making for contact tracing are outlined.

\section{Background}

With increasing numbers of passengers travelling internationally by air the potential risk of introduction and spread of infectious diseases by travellers increases. In 2009, the global airport traffic reported $4.796 \times 10^{9}$ passengers arriving and departing from 1,354 airports located in 171 countries worldwide, with passengers on international flights accounting for 42 percent [1]. Almost 800 million passengers are carried on national/ international flights annually within the European Union (EU) alone [2].

The outbreak of SARS in 2003 and pandemic influenza $A\left(\mathrm{H}_{1} \mathrm{~N}_{1}\right)$ in 2009 illustrated how infectious diseases can suddenly appear, spread, and threaten the health, economy and social lives of citizens even in countries that are not or not yet affected by the epidemic itself. When passengers and/or crew members become exposed to an infectious or potentially infectious person during a flight, early recognition of disease and coordinated risk assessment among the affected countries is needed to initiate appropriate public health response without unnecessarily alarming the public and disrupting air traffic.

There are legal obligations for the member states of the World Health Organization (WHO) to report events of public health concern in accordance with the International Health Regulations (IHR) [3] and for the Member States of the EU to provide information to the Community Network in accordance to the Decision No 2119/98/EC [4]. However, very limited international guidance exists for the public health management of infectious diseases related to air travel, both aboard aircrafts and at airports [5]. Existing international guidance, e.g. the WHO international guidelines for the control of tuberculosis [6], does not necessarily reflect the epidemiologic situation in the individual EU Member States, while the national guidelines, e.g. for meningococcal diseases [7], are frequently inconsistent.

In order to assist national public health authorities in EU Member States in the evaluation of risks related to the transmission of various infectious agents on board aircrafts and to help in the decision on the most appropriate, operationally possible public health measures for containment, e.g. on whether or not to contacttrace air travellers and crew in case of exposure, the European Centre for Disease Prevention and Control (ECDC) initiated in 2007 the project Risk Assessment Guidance for Infectious Diseases transmitted on Aircraft (RAGIDA) [8].

The RAGIDA project consists of two parts: (i) a systematic review of the literature of documented past events of infectious disease transmission on aircrafts, guidance documents and expert interviews assessing casebased information on events (produced by the Robert Koch Institute, Germany in response to an ECDC open call for tender 0J/2007/06/20-PROC/2007/009) [8], and (ii) a series of disease-specific guidance documents 
produced by external disease-specific expert panels [9] on which this article will mainly focus. This guidance does not address contacts at the airport or occurring during transit.

\section{Methods}

Part I: Systematic review and expert interviews In the first part of the RAGIDA project a systematic review of over 3,700 peer-reviewed articles and grey literature was performed for the following 12 infectious diseases: tuberculosis, influenza, SARS, invasive meningococcal disease, measles, rubella, diphtheria, Ebola and Marburg haemorrhagic fevers, Lassa fever, smallpox and anthrax. The aim was to evaluate the exact circumstances that led to the transmission of these infectious diseases on board aircrafts. For peerreviewed publications, PubMed and the database of the German Institute of Medical Documentation and Information (DIMDI) were searched, using the following two combinations of search terms: (aircraft OR airplane OR flight $O R$ flight crew $O R$ air travel $O R$ airline $O R$ air passenger) AND (epidemiology OR microbiology OR transmission), (aircraft OR airplane OR flight OR flight crew $O R$ air travel $O R$ airline $O R$ air passenger) AND (infectious).

Grey literature was searched in ProMed using the search terms 'airline OR air travel OR air passenger'. In addition, general guidelines on risk assessment and management were systematically searched from international aviation boards, the Airport Council International (ACI), International Air Transport Association (IATA) and International Civil Aviation Organisation (ICAO) and several national and international public health agencies such as the WHO, the United States Centers for Disease Control and Prevention, Health Canada, the Health Protection Agency in the United Kingdom and the Robert Koch Institute in Germany. Standardised questionnaires were used to interview an international group of experts to collect case-based information on events.

Contacts were defined as persons with relevant exposure to an infectious or potentially infectious index case. The credibility of an exposure was assessed by referring to event-specific factors such as pathogen, infectiousness of the index case, infectious period, availability of information on on-board exposure, possible alternative exposures, and risk factors for infection. The evidence of on-board transmission was assessed for each event according to a set of established criteria. These criteria took into account the validity and relevance of diagnostic tests (index case(s)/contacts), the validity and relevance of information for exposures or alternative exposures of contacts, and the susceptibility of contacts. Evidence for transmission was graded into four categories: high, probable, possible and none. If no transmission was concluded, the level of evidence for non-transmission was assessed using the proportion of the successfully traced contacts among all susceptible contacts on board the flight. The evidence was assessed as low if the proportion was smaller than $35 \%$; medium if the proportion was between $35 \%$ and $75 \%$, and high if the proportion was larger than $75 \%$.

\section{Part II: Disease-specific guidance}

Within the second part of the RAGIDA project, the production of a series of operational guidance documents for assisting in the evaluation of risk for transmission of diseases was initiated. In June 2009, ECDC convened the first RAGIDA disease-specific expert meeting that focused on tuberculosis, SARS and invasive meningococcal infections. In 2010 a second meeting followed that concentrated on measles, rubella, Ebola and Marburg haemorrhagic fevers, Lassa fever, smallpox and anthrax.

For both meetings, small, multidisciplinary diseasespecific expert panels were established. The participants were selected to include representatives of national public health authorities, particularly those with experience in the investigation and follow-up of incidents involving infectious diseases in travellers, European and international experts for the disease(s) under investigation, experts in microbiology and mathematic modelling, and representatives of the ECDC, the European Commission and the WHO International Health Regulations Coordination Programme. No conflicts of interest were declared by any of the participants.

Evidence obtained included the review of the published literature by disease related to air travel, the review of data on air travellers obtained from national public health authorities (from RAGIDA part I), and expert opinions from the members of the expert panel. Experts discussed basic elements of the Scottish Intercollegiate Guidelines Network (SIGN) approach for developing guidelines [10] and reviewed the evidence base taking into account the available scientific evidence for disease transmission as well as other relevant aspects such as disease severity, the potential for public health intervention, and availability of treatment.

Each disease-specific chapter contains a short literature review, outlines an approach for contact tracing including an algorithm and a template for questions and answers.

\section{Results}

Part I: Systematic review and expert interviews The available information published in peer-reviewed journals was very limited for most of the diseases for which only a few on-board transmission events were described, limiting the power for evidence-based decision making. With the exception of tuberculosis no international guidance for contact tracing was identified $[7,11,12]$. A detailed report of this first part of the project has been published [8]. 
Part II: Disease-specific guidance

Overall the expert panels agreed that for each of the diseases contact tracing should be recommended only after careful risk assessment. Contact tracing was considered as reasonable if the probability of an infectious disease causing a secondary infection and/or further spread in the population was high in conjunction with an assessment that the impact on human health in terms of an adverse outcome (the scale of harm caused by the infectious threat in terms of morbidity and mortality) was also high. Several additional factors were identified that influence the decision making regarding contact tracing.

\section{Factors that affect the probability of}

disease transmission on board aircrafts

The probability that a certain infectious disease is transmitted on board an aircraft depends on characteristics of the causative agent and the host, and on environmental factors. These include:

- infectivity of the index case during the flight in the symptomatic or pre-symptomatic stage, taking into account epidemiological attributes such as Ro, period of shedding, infectiousness period, mode of transmission, as well as signs and symptoms of disease;

- susceptibility of the passengers, considering their level of natural immunity and vaccination status;

- effectiveness of exposure, depending on proximity to the index case, duration of exposure as well as the technical specifications of the airplane and the quality of the cabin air.

Factors that affect the impact on human health The impact on human health, the scale of harm that a certain infectious disease causes in terms of morbidity and mortality, depends on characteristics of the pathogen and the host, and on the available means for detection and intervention. The relevant factors include:

- pathogen-specific attributes for disease manifestation such as virulence, resistance pattern and case fatality;

- underlying condition associated with severity, considering compromised immune system, comorbidity or pregnancy;

- means for detection and possibilities for diagnosis, taking into account the availability and reliability of diagnostic tests;

- effectiveness of intervention, e.g. availability of prophylaxis and/or treatment.

\section{Factors that influence the decision on} contact tracing

In addition to the probability of transmission and the impact on human health, there are several additional factors that influence the decision making regarding contact tracing, such as:

- susceptibility of the passengers for the disease, taking into account the level of natural immunity and the vaccine coverage in the population of the countries of origin and destination;

- the maximum incubation period, i.e. the time period during which it is possible to intervene with public health measures; contact tracing at a later time could be initiated for scientific purposes;

- ethical aspects, e.g. whether treatment is available or whether containment and/or mitigation measures are acceptable for the contacts;

- means for response, i.e. the public health actions taken after identification of infected individuals, the options that can be offered to the infected individuals identified by contact tracing;

- alternative actions instead of contact tracing such as risk communication including leaflets for passengers of the flight and information on airports;

- media coverage and public attention;

- political sensitivities in the involved countries;

- available resources.

\section{Discussion}

In a globalised world, the risk for transmission and spread of infectious diseases through travel and trade needs to be addressed. In terms of passenger numbers, Europe has four of the eleven airports receiving the highest passenger numbers worldwide: London, Paris, Frankfurt and Madrid. Each of them receive more than 50 million passengers a year (with the larger proportion of passengers on international flights) $[1,2]$, some of whom are likely to have or incubate infectious diseases. Airline cabins, as confined spaces, may provide an environment for disease transmission. There is some evidence from studies examining microbial contaminants in cabin air, that suggest air quality in an airline cabin is better than in most buildings [13-15] and most other means of public transportation (e.g. buses, trains, subways). Most modern airplanes operate a ventilation system with laminar air flow with exchange rates of 20 air exchanges per hours during cruising. Before re-entering the cabin, the air is filtered through a set of high-efficiency particulate air (HEPA) filters, which remove at least $99.97 \%$ of airborne particles between 0.1 and $0.3 \mu \mathrm{m}$ in diameter and $100 \%$ of particles larger than $0.3 \mu \mathrm{m}$ in diameter. However, when an aircraft is parked at the gate with the engines off for more than 30 minutes with passengers on board, adequate cabin ventilation should be ensured [16].

According to the IHR which legally bind 194 States worldwide, events of disease transmission among passengers on international flights require notification to the WHO [3]. Member States of the EU must further provide information on such cases through the appropriate designated structures and/or authorities in a timely manner to allow an effective joint response of the affected countries [4].

Assessing the risk of transmission of infectious diseases on board an aircraft is not always easy and often has to rely on individual expert opinion. The available 
evidence is limited and assessing the publicly available evidence retrieved from the literature/grey literature is challenging. For most of the infectious diseases only a small number of studies are available on a limited number of events. The majority of the studies are observational, lack an appropriate control group and do not control for biases. In most of the reported studies the proportion of passengers (contacts) successfully traced and followed up is small, and for diseases with a long incubation period such as tuberculosis, asymptomatic passengers are often not followed up long enough to document seroconversion. For diseases with a high proportion of asymptomatic or mild cases or with an atypical presentation, cases are less likely to be detected because diagnostic tests are less likely to be performed. In addition, studies not showing transmission or disease outcome are less likely to be published (publication bias).

The decision on public health action and contact tracing has to be made fast and is influenced by several factors that differ between countries, such as the available resources, the purpose of contact tracing, its feasibility and the perception of the risk of the disease when evidence is lacking or when media attention or political pressure is high. Contact tracing requires significant resources in terms of manpower, money, and time. The amount of resources needed further depends on the objective of the tracing, e.g. whether it is done to initiate disease containment measures, disease mitigation measures, to delay the spread of the disease or to eradicate the disease. Only a limited number of studies are available on the cost-effectiveness of contact tracing in this regard. In the case of tuberculosis several studies indicate that the costs are high and the outcome is poor $[17,18]$. It must also be considered that adequate contact tracing in resource-poor countries may come at the expense of other more effective health measures [18]. Contact tracing is often complicated when passenger information is lacking. Aircraft manifests are not standardised across airlines and passenger lists are rarely kept for more than 48 hours. Legal matters and data protection issues could hamper the exchange of information between countries and organisations. Communication and coordination between the different national authorities can be complex and the proportion of contacts that can be successfully traced is often rather small $[19,20]$.

Finally the perception of a risk plays a crucial role in its assessment and the decision for contact tracing. Assessments are influenced not only by the societal environment in which events occur and decisions are being made, but also by politics and the economic situation in a country. An infectious disease assessed at low risk, for instance, can have a significant economic and political impact in a certain context.

\section{Conclusions}

Considering the lack of published data available on evaluating the risk of transmission of most infectious agents on board aircrafts, and taking into account the key factors that influence the decision making, the RAGIDA guidance provides a viable evidence-based tool for public health authorities determining triggers and making decisions on whether to undertake contact tracing in air travellers or crew. These guidance documents may be adapted to the local situation, national and international regulations or preparedness plans. To improve the evidence base for contact tracing and to conclude on the cost-effectiveness of this public health intervention, information on the outcome of disease events during air travel needs to be collected continuously as initiated by this project.

\section{Acknowledgements}

\section{Participants for RAGIDA Part 1}

Robert Koch Institute: Karl Schenkel, Rachel Amorosa, Inge Mücke, Valentina Dias-Ferrao, Michaela Diercke, Gérard Krause, Tim Eckmanns.

Interviewed experts: Preben Aavitsland, Franz Allerberger, Paulo M. Alves, Peter Andersen, Thomas Bettes, Dounia Bitar, Bonita Brodhun, Udo Buchholz, Silke Buda, Neville Byrne, Marty Cetron, Jacques Chemardin, Miguel Dávila Cornejo, Annette Dietrich, Suzanne Cotter, Susan Courage, Roel Coutinho, Agnes Csohan, Jean-Claude Desenclos, Jozef Dlhý, Anou Dreyfus, Sabine Erne, Anthony Evans, Christina Frank, David Gamper, Helen Giamarella, Miguel Mínguez Gonzalo, Rene Gottschalk, Walter Haas, Danielle HansenKoenig, Wiebke Hellenbrand, Pierrette Huberty-Krau, Ansa Jordaan, Irena Klavs, Jan Klika, Judith Koch, Henry Kong, Ichiro Kurane, Kuulo Kutsar, Irina Lucenko, Tomohiko Makino,Patrick Mathys, Karen Marineau, Tanya Melillo Fenech, Kore Molbak, Maria Jose Sierra Moros, Ion Morrison, Howard Njoo, Joan O’Donnell, Darina O'Flanagan, Rose Ong, Maria Teresa Avilez Paixao, Androula Pavli, Gabriele Poggensee, Jelena Rjabinina, Petri Ruutu, Daniel Sagebiehl, Stefania Salmaso, Kirsten Schubert, Anette Siedler, René Snacken, Gianfranco Spiteri, Klaus Stark, Robert Steffen, Arnaud Tarantola, Claude Thibeault, Thomas Tsang, Rolanda Valinteliene, Anneke van den Hoek, Cliff Webster, Brita Winje, Anne Witschi.

\section{Participants for RAGIDA Part 2}

Tuberculosis expert group: Ibrahim Abubakar (chair), Jacques Chemardin, Annick Darrieu-Merlou, José Figueroa, Peter Helbling, Jean-Paul Klein, Vladimir Prikazsky, Gerard De Vries.

SARS expert group: Philippe Barboza, Christian Drosten, Tim Eckmans (chair), Celine Gossner, Helge Hollmeyer, Masja Straetemans, Sylvie Van der Werf, Peter White.

Meningococcal disease expert group: Suzanne Cotter (chair), Ida Czumbel, Paolo D'Ancona, Wiebke Hellenbrand, Sigrid Heuberger, Isabelle Parent du Chatelet, Pawel Stefanoff, Lena de Vries.

Measles and rubella expert group: Kevin Brown, Donato Greco (chair), Susan Hahné, Peter Kreidl, Claude Muller, Mark Muscat, Darina O'Flanagan, Anette Siedler.

Viral haemorrhagic fevers expert group: Roberta Andraghetti, Ray Arthur, Andreas Gilsdorf, Stephan Günther, Michel van Herp, Katrin Leitmeyer, Dilys Morgan (chair), Aura Timen, Maggie Tomlinson.

Smallpox and anthrax expert group: Franz Allerberger (chair), Agoritsa Baka, Tim Brooks, Roland Grunow, Sabina Lyson, Vincenzo Puro, Sybille Rehmet, Anna Swiatecka, Alexander Theus. 


\section{References}

1. Airports Council International (ACl). ACl Annual World Airport Traffic Report 2009. Montreal: ACl; July 2009. Available from: http://www.aci.aero/cda/aci_common/display/main/aci_ contento7 c.jsp?zn=aci\&cp=1-6-43-3647^2003 $666 \_2$

2. Eurostat. Europe in figures. Eurostat yearbook 2010. Luxembourg: Eurostat; 2010. Available from: http://epp. eurostat.ec.europa.eu/cache/ITY_OFFPUB/KS-CD-10-220/EN/ KS-CD-10-220-EN.PDF

3. World Health Organization (WHO). International Health Regulations (2005). 2nd ed. Geneva: WHO; 2008. Available from: http://whqlibdoc.who.int/ publications/2008/9789241580410_eng.pdf

4. European Commission. Decision No 2119/98/EC of the European Parliament and of the Council of 24 September 1998 setting up a network for the epidemiological surveillance and control of communicable diseases in the Community. Official Journal of the European Union. Luxembourg: Publications Office of the European Union; 3 Oct 1998. L 268. Available from: http://eur-lex.europa.eu/LexUriServ/LexUriServ.do?uri= CELEX:31998D2119:EN:HTML

5. Gaber, W. Goetsch U, Diel R, Doerr HW, Gottschalk R. Screening for infectious diseases at international airports: the Frankfurt model. Aviat Space Environ Med. 2009;80(7):595-600.

6. Hoek, M, Hanquet G, Heuberger S, Stefanoff P, Zucs P, Ramsay $M$, et al. A European survey on public health policies for managing cases of meningococcal disease and their contacts. Euro Surveill. 2008;13(10):pii=8060. Available from: http:// www.eurosurveillance.org/ViewArticle.aspx?Articleld=8060

7. World Health Organization (WHO). Tuberculosis and air travel: guidelines for prevention and control. 3rd ed. Geneva: WHO; 2008. Available from: http://www.who.int/tb/ publications/2008/WHO_HTM_TB_2008.399_eng.pdf

8. European Centre for Disease Prevention and Control (ECDC). Risk assessment guidelines for infectious diseases transmitted on aircraft. Stockholm, ECDC: Jun 2009. Available from: http:// www.ecdc.europa.eu/en/publications/publications/0906 ter_risk_assessment_guidelines_for_infectious_diseases_ transmitted_on_aircraft.pdf

9. European Centre for Disease Prevention and Control (ECDC). Risk assessment guidelines for diseases transmitted on aircraft. Part 2: Operational guidelines for assisting in the evaluation of risk for transmission by disease. Stockholm, ECDC; Nov 2009. Available from: http://ecdc.europa.eu/en/ publications/Publications/0911_GUI_Risk_Assessment Guidelines_for_Diseases_Transmitted_on_Aircraft.pdf

10. Scottish Intercollegiate Guidelines Network (SIGN). SIGN 50: a guideline developer's handbook. Chapter 7: Forming guideline recommendations. Edinburgh: SIGN; Jan 2008. http://www. sign.ac.uk/pdf/sign50.pdf

11. World Health Organization (WHO). Tuberculosis and air travel: Guidelines for prevention and control. Geneva: WHO; 1998. Available from: http://www.emro.who.int/stb/media/ pdf/98_256.pdf

12. World Health Organization (WHO). Tuberculosis and air travel: Guidelines for prevention and control. 2nd ed. Geneva: WHO; 2006. Available from: http://whqlibdoc.who.int/hq/2006/ WHO_HTM_TB_2006.363_eng.pdf

13. Nagda NL, Koontz MD. Review of studies on flight attendant health and comfort in airliner cabins. Aviat Space Environ Med. 2003;74(2):101-9.

14. Dechow M, Sohn H, Steinhanses J. Concentrations of selected contaminants in cabin air of airbus aircrafts. Chemosphere. 1997;35(1-2):21-31.

15. Wick RL Jr, Irvine LA. The microbiological composition of airliner cabin air. Aviat Space Environ Med. 1995;66(3):220-4.

16. Mangili A, Gendreau MA. Transmission of infectious diseases during commercial air travel. Lancet. 2005;365(9463):989-96.

17. Vassiloyanakopoulos A, Spala G, Mavrou E, Hadjichristodoulou C. A case of tuberculosis on a long distance flight: the difficulties of the investigation. Euro Surveill. 1999;4(9): pii=83. Available from: http://www.eurosurveillance.org/ ViewArticle. aspx?Articleld $=83$

18. McFarland JW, Hickman C, Osterholm M, MacDonald KL. Exposure to Mycobacterium tuberculosis during air travel. Lancet. 1993;342(8863):112-3.

19. Abubakar I. Tuberculosis and air travel: a systematic review and analysis of policy. Lancet Infect Dis. 2010;10(3): 176-83.

20. Kornylo-Duong K, Kim C, Cramer EH, Buff AM, RodriguezHowell D, Doyle J, et al. Three air travel-related contact investigations associated with infectious tuberculosis, 20072008. Travel Med Infect Dis. 2010;8(2):120-8. 